\title{
Influence of intense coherent electromagnetic radiation on several types of radioactive decay
}

\author{
S.N. Andreev ${ }^{2}$, E.V. Barmina ${ }^{2}$, A.K. Kaminsky ${ }^{1}$, S.N. Sedykh ${ }^{1}$, G.A. Shafeev ${ }^{2}$, \\ I.A. Shcherbakov ${ }^{2}$, A.V. Simakin ${ }^{2}$, V.I. Stegailov ${ }^{1}$, S.I. Tyutyunnikov ${ }^{1}$ \\ ${ }^{1}$ Joint Institute for Nuclear Research, Dubna, Russia, snsed@yandex.ru \\ ${ }^{2}$ Prokhorov General Physics Institute RAS, Moscow, Russia
}

\section{Optical laser radiation}

Many configurations have been proposed to use highpower laser radiation for acceleration of the charged particles [1]. Required power density for such experiments varied from $10^{18} \mathrm{~W} / \mathrm{cm}^{2}$ up to $10^{20} \mathrm{~W} / \mathrm{cm}^{2}$. For example, $\mathrm{X}$-rays of $\mathrm{MeV}$ energy have been produced by accelerated plasma electrons when laser beam with intensity of $5 * 10^{18} \mathrm{~W} / \mathrm{cm}^{2}$ was focused on tantalum sheet. Energy of these X-rays was high enough to initiate a set of nuclear reactions, which have been registered experimentally [2].

We investigate an influence of coherent electromagnetic radiation on different types of radioactive decay. Our experiments indicate that some nuclear reactions can be initiated even at laser beam intensity about $1 * 10^{12} \mathrm{~W} / \mathrm{cm}^{2}$. The key feature is the presence of metallic (chemically inactive $\mathrm{Au}$ as a rule) nanoparticles. In plasmon resonance regime the wave field near nanoparticles can be concentrated up to the necessary level. Layout of the typical experiment is shown on fig. 1.

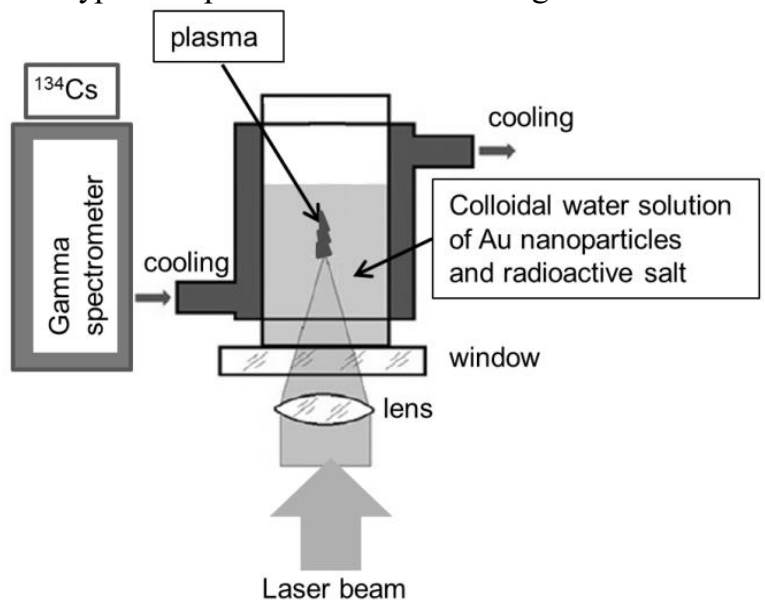

Fig. 1. Layout of the experiment on irradiation of water solution of the radioactive salt by the medium-power laser in presence of the metallic nanoparticles

We use several types of optical and near-infrared lasers - $\mathrm{Cu}$ vapor, femtosecond Ti:sapphire, 90-ps Nd:YAG, 350-ps Nd:YAG, 10-ns Nd:YAG, Their pulse energy varied from $0.1 \mathrm{~mJ}$ up to $40 \mathrm{~mJ}$, repetition rate varied from $10 \mathrm{~Hz}$ up to $20 \mathrm{kHz}$. Power density in focal region without influence of the nanoparticles varied from $1 * 10^{11}$ $\mathrm{W} / \mathrm{cm}^{2}$ up to $1 * 10^{12} \mathrm{~W} / \mathrm{cm}^{2}$. Nanoparticles being used in our experiments are produced by laser ablation by metal target in the water.

We have observed partial $\mathrm{Hg}$ transmutation into $\mathrm{Au}$ inside heavy water $\mathrm{D}_{2} \mathrm{O}$ [3]. Laser irradiation in presence of $\mathrm{Au}$ nanoparticles initiates the decay of ${ }^{232} \mathrm{Th}$ [4]. Very exciting result was a significant (up to 50\%) decrease of gamma-activity of ${ }^{238} \mathrm{U}$ and ${ }^{235} \mathrm{U}$ series after laser irradiation in presence of beryllium nanoparticles [5].

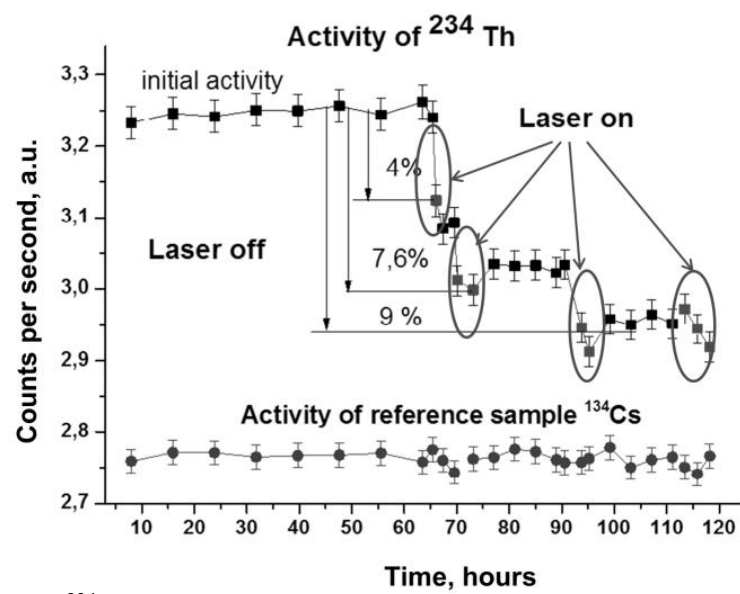

Fig. 2. ${ }^{234}$ Th activity during several sessions of laser irradiation of $\mathrm{UO}_{2} \mathrm{Cl}_{2}$ water solution in presence of $\mathrm{Au}$ nanoparticles as the laser wave concentrators. ${ }^{134} \mathrm{Cs}$ is a reference sample

Figure 2 presents the results of long-time measurement of the ${ }^{234}$ Th activity during several sessions of laser irradiation. Each session decrease the activity. Effect of each next irradiation is less than the previous one due to fragmentation of nanoparticles and their escape from the plasmon resonance.

\section{Microwave radiation}

There are some similar experiments in microwave and radiofrequency regions. $3 \mathrm{GHz}$ radiation caused increasing the ${ }^{51} \mathrm{Cr}$ activity at $1 \%$ [6]. $0.07 \%$ of the ${ }^{137} \mathrm{Cs}$ activity growth was registered during irradiation of the sample by $4.1 \mathrm{MHz}$ and $1.55 \mathrm{MHz}$ radiation with a maximum power of $50 \mathrm{~kW}$ [7]. Our experiment using $30 \mathrm{GHz}$ FEM shows about $1.5 \%$ variation of the ${ }^{152} \mathrm{Eu}$ activity [8]. In all these experiments there were no special measures to increase the power density.

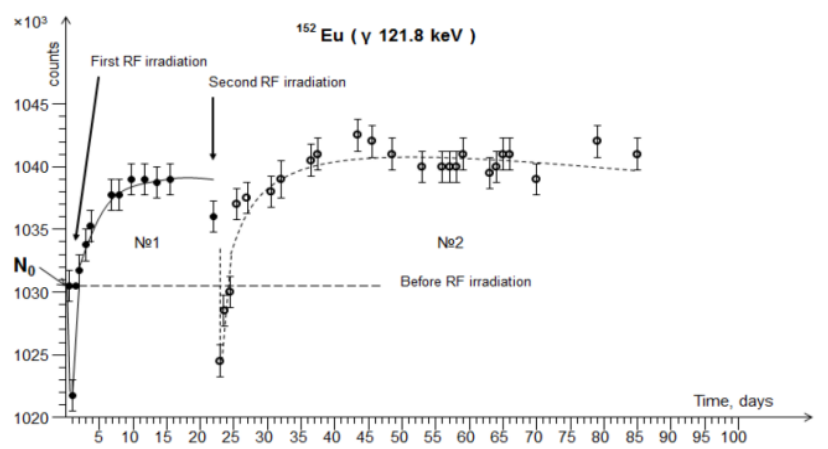

Fig. 3. Activity of the ${ }^{152} \mathrm{Eu}$ during two irradiations by $15 \mathrm{MW} 30 \mathrm{GHz}$ JINR-IAP FEM oscillator 


\section{Field concentrators in microwave experiment}

We assume that all changes in the rate of the radioactive decay were caused by the charged particles accelerated in the plasma, produced in focal region in presence of field concentrators (metal nanoparticles). We have in mind that the power density about $1 * 10^{18} \mathrm{~W} / \mathrm{cm}^{2}$ is enough to produce some types of nuclear reaction without special field concentrators. Estimation of the maximal energy of the proton inside the electromagnetic wave with wavelength $\lambda$ and intensity $I$ can be done by [9]:

$$
E_{\max }=3.6^{*} \lambda \sqrt{I / 10^{18}}
$$

where $E_{\max }$ is in $\mathrm{MeV}, \lambda$ in microns, $I$ in $\mathrm{W} / \mathrm{cm}^{2}$. Indeed, the wave with length of 1 micron and intensity of $1 * 10^{18} \mathrm{~W} / \mathrm{cm}^{2}$ can accelerate protons up to $3.6 \mathrm{MeV}$, which is more than the threshold of many nuclear reactions. In our experiments with power densities of $1 * 10^{12} \mathrm{~W} / \mathrm{cm}^{2}$ the presence of field concentrators (nanoparticles) can increase the field intensity up to $10^{5}$ and (nanoparticles dimers) to $10^{6}$ times.

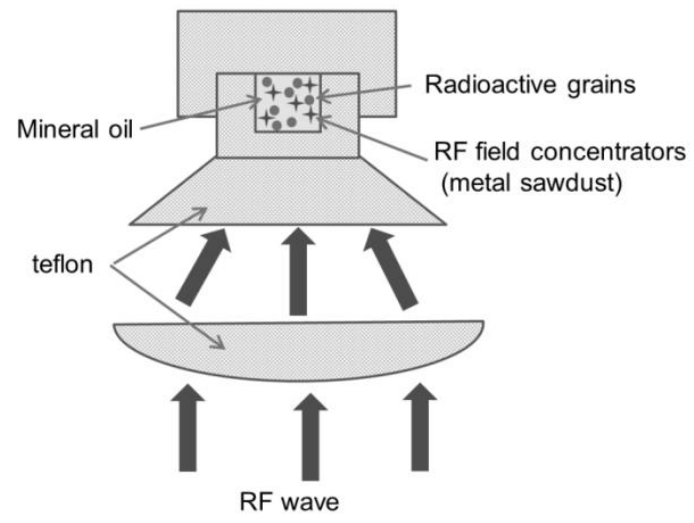

Fig. 4. Proposed layout of the experiment on the RF irradiation of the radioactive sample. Metal sawdust should be the RF field concentrators

Let us estimate the necessary rate of field concentration in microwave region. For wavelength of $1 \mathrm{~cm}$ $\left(10^{4}\right.$ microns) the desired proton energy of $3.6 \mathrm{MeV}$ requires the field intensity of $1 * 10^{10} \mathrm{~W} / \mathrm{cm}^{2}$. The diffraction limits the area of focal spot by $1 \mathrm{~cm}^{2}$, so we need or $10 \mathrm{GW}$ radiation source, or $10 \mathrm{MW}$ radiation source and $1 * 10^{3}$ times field concentrators. For microwave region the role of the field concentrators can play metal sawdust. The layout of proposed experiment is shown on fig. 4.

\section{References}

1. Krushelnick K., Najmudin Z. and Dangor A.E. Particle acceleration using intense laser produced plasmas // Laser Phys. Lett. 2007. V. 4, No. 12 P. 847-862.

2. Schwoerer H., P. Gibbon P., S. Düsterer S. et al. MeV $\mathrm{X}$ rays and photoneutrons from femtosecond laser-produced plasmas // Phys. Rev. Lett. 2001. V. 86, No. 11 P. 2317-2320.

3. Shafeev G.A., Bozon-Verduraz F. and Robert M. Experimental evidence of transmutation of $\mathrm{Hg}$ into $\mathrm{Au}$ under laser exposure of $\mathrm{Hg}$ nanodrops in $\mathrm{D}_{2} \mathrm{O} / /$ Physics of Wave Phenomena 2007. V. 15, No. 13 P. 131-136.

4. Simakin A.V. and Shafeev G.A. Initiation of nuclear reactions under laser irradiation of metal nanoparticles in the presence of Thorium aqua ions // Physics of Wave Phenomena 2008. V. 16, No. 4 P. 268-274.

5. Barmina E.V., Sukhov I.A., Lepekhin N.M. et al. Application of copper vapour lasers for controlling activity of uranium isotopes // Quantum Electronics 2013. V. 4, No. 6. P. 581-596.

6. Rybasov A.G., Ryzhkov V.A., Len' N.A. and Sulakhshin A.S. Patent of Russian Federation No 2100858 (C1) G21F 9/00 1995 (in Russian).

7. Reiss H.R. Observation of the acceleration by an electromagnetic field of nuclear beta decay // A Letters Journal Exploring the Frontiers of Physics EPL 2008. V. 81 P. 42001.

8. Golubykh S.M., Gons Z., Kalinnikov V.G. et al. Influence of high-power pulse RF irradiation on several types of Betadecay // Proceedings of $9^{\text {th }}$ Intern. Workshop "Strong microwaves and terahertz waves: sources and applications" 2014. P. 61-62.

9. Maksimchuk A., Flippo K., Krause H. et al. High-energy ion generation by short laser pulses // Plasma Phys. Rep. 2004. V. 30 No 6 P. 473-495. 